\title{
5,6-DIHYDRO-[1,2,4]TRIAZOLO[1,5-c]QUINAZOLINES. MESSAGE 4. SPIROCOMPOUNDS WITH [1,2,4]TRIAZOLO[1,5-c] QUINAZOLINES MOIETIES. THE SYNTHESIS AND SPECTRAL CHARACTERISTICS
}

\author{
S.V.Kholodnyak ${ }^{1}$, K.P.Schabelnyk ${ }^{1}$, O.Yu.Voskoboynik ${ }^{1}$, O.M.Antypenko ${ }^{1}$, \\ S.I.Kovalenko ${ }^{1}$, V.O.Palchykov ${ }^{2}$, S.I.Okovyty ${ }^{2}$, S.V.Shishkina ${ }^{3}$ \\ ${ }^{1}$ Zaporizhzhya State Medical University, \\ 26, Mayakovsky Ave., Zaporizhzhya, 69035, Ukraine \\ ${ }^{2}$ Dnipropetrovsk National University \\ ${ }^{3}$ SSI "Institute for Single Crystals", National Academy of Sciences of Ukraine
}

Key words: 2'-aryl-6'H-spiro[1,2,4]triazolo[1,5-c]quinazolines; synthesis; spectral characteristics

\begin{abstract}
The present article describes the synthesis of novel spiro-condensed [1,2,4]triazolo[1,5-c]quinazolines. [2-(3-Aryl-

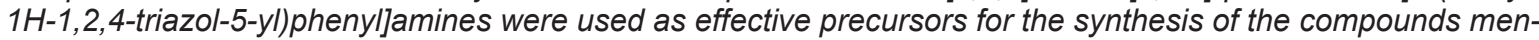
tioned above. The experimental data have shown that the reaction of the initial anilines with cycloalkanones (cyclopentanone, cyclohexanone) allowed to obtain products of binucleophilic addition, namely spiro-condensed compounds with $[1,2,4]$ triazolo[1,5-c]quinazolines moieties. The initial anilines also readily react with a conformationally rigid bicyclo[2.2.1]heptan-2-one yielding the corresponding spiroderivatives, whereas the reaction with camphor and menthone has failed due to the steric hindrance. It has been found that [5+1]-cyclocondensation of the initial anilines with heterocyclonones (1-R-piperidone-4, dihydrothiophene-3(2H)-one, dihydro-2H-pyran-4(3H)-one, dihydro-2H-thiopyran-3(4H)-one) proceeds without peculiarities and with formation of the corresponding 2'-aryl-6' $\mathrm{H}$-spiro[1,2,4]triazolo[1,5-c]quinazolines. The reaction with 5-R-1H-indole-2,3-dione (isatine) and its $N$-substituted derivatives also proceeds without any peculiarities with formation of aryl-2'-aryl6'H-spiro[(indol-3,5'-[1,2,4]triazolo[1,5-c]quinazolines] with high yields. The purity of the compounds obtained has been proven by the LC-MS (APCI) method, their structures have been confirmed by the complex of physicochemical methods, including ${ }^{1} \mathrm{H}$ and ${ }^{13} \mathrm{C} N M R$, IR-, MS-(EI) - spectrometry and the X-ray study. The peculiarities of ${ }^{1} \mathrm{H}$ and ${ }^{13} \mathrm{C} N M R$-spectra of the compounds synthesized are discussed. It has been shown that signals of NH-protons in the ${ }^{1} \mathrm{H} N M R$-spectrum and $\mathrm{C}-5$ ' in the ${ }^{13} \mathrm{C}$ NMR-spectrum are characteristic for the compounds synthesized.
\end{abstract}

5,6-ДИГІДРО-[1,2,4]ТРИАЗОЛО[1,5-с]ХІНАЗОЛІНИ. ПОВІДОМЛЕННЯ 4. СПІРОПОХІДНІ 3 [1,2,4]ТРИАЗОЛО[1,5-с] ХІНАЗОЛІНОВИМ ФРАГМЕНТОМ. СИНТЕЗ ТА СПЕКТРАЛЬНІ ХАРАКТЕРИСТИКИ

С.В.Холодняк, К.П.Шабельник, О.Ю.Воскобойнік, О.М.Антипенко, С.І.Коваленко, В.О.Пальчиков, С.І.Оковитий, С.В.Шишкіна

Ключові слова: 2-арил-6'Н-спіро[1,2,4]триазоло[1,5-с]хіназоліни; синтез; спектральні характеристики Описано синтез нових спіроконденсованих [1,2,4]триазоло[1,5-с]хіназолінів. [2-(3-Арил-1Н-1,2,4-триазол5-іл)френіл]аміни були використані в якості ефрективних прекурсорів для синтезу згаданих вище сполук. Зәідно з експериментальними даними реакція вихідних анілінів з циклоалканонами (циклопентаноном, циклогексаноном) дозволила одержати продукти бінуклеофрільного приєднання, а саме спіроконденсовані сполуки з [1,2,4]триазоло[1,5-с]хіназоліновим фррагментом. Вихідні аніліни також реагують з конформаційно жорстким біцикло[2.2.1]гептан-2-оном, що веде до формування відповідних спіропохідних, в той же час реакцію з камфророю та ментоном провести не вдалось внаслідок стеричних ускладнень. Встановлено, що [5+1]-циклоконденсація вихідних анілінів з гетероцикланонами (1-R-піперидоном-4, дигідротіофрен-3(2H)-оном, дигідро-2Н-піран-4(3Н)-оном, дигідро-2Н-тіопіран-3(4H)-оном) перебігає без особливостей з утворенням відповідних 2'-арил-6'Н-спіро[1,2,4]триазоло[1,5-с]хіназолінів. Також без особливостей перебігає реакція з 5-R-1H-індол-2,3-діоном (ізатином) та його N-заміщеними похідними з утворенням арил-2'-арил-6' Н-спіро[(індол-3, 5'-[1,2,4]триазоло[1,5-с]хіназолінів] з високими виходами. Чистота синтезованих сполук була доведена методом LC-MS (APCI), їх будову підтверджено комплексом фрізико-хімічних методів, зокрема ${ }^{1} \mathrm{H}$ ma ${ }^{13} \mathrm{C}$ ЯMP, I4-, MC-(ЕУ)-спектрометрично та за допомогою рентгеноструктурного дослідження. Особливості ${ }^{1} \mathrm{H}$ ma ${ }^{13} \mathrm{C}$ ЯМР-спектрів синтезованих сполук були обговорені. Показано, що для синтезованих сполук характеристичними є сигнали $\mathrm{NH}$-протонів у ${ }^{1} \mathrm{H}$ ЯМР спектрах та С-5' в ${ }^{13} \mathrm{C}$ ЯМР-спектрах.

5,6-ДИГИДРО-[1,2,4]ТРИАЗОЛО[1,5-с]ХИНАЗОЛИНЫ. СООБЩЕНИЕ 4. СПИРОПРОИЗВОДНЫЕ С [1,2,4] ТРИАЗОЛО[1,5-С]ХИНАЗОЛИНОВЫМ ФРАГМЕНТОМ. СИНТЕЗ И СПЕКТРАЛЬНЫЕ ХАРАКТЕРИСТИКИ С.В.Холодняк, К.П.Шабельник, А.Ю.Воскобойник, А.Н.Антипенко, С.И.Коваленко, В.А.Пальчиков, С.И.Оковытый, С.В.Шишкина

Ключевые слова: 2-арил-6'H-спиро[1,2,4]триазоло[1,5-с]хиназолины; синтез; спектральные характеристики

В представленной статье описан синтез новых спироконденсированных [1,2,4]триазоло[1,5-с]хиназолинов. [2-(3-Арил-1Н-1,2,4-триазол-5-ил)френил]амины были использованы в качестве эфффективных прекурсоров для синтеза упомянутых выше веществ. Согласно экспериментальных данных реакция исход- 
ных анилинов с циклоалканонами (циклопентаноном, циклогексаноном) позволила получить продукты би-нуклеофильного присоединения, а именно спироконденсованные соединения с [1,2,4]триазоло[1,5-c] хиназолиновым фррагментом. Исходные анилины также реагируют с конформационно жестким бицикло[2.2.1]еептан-2-оном, что приводит к формированию соответствующих спиропроизводных, в то же самое время реакцию с камфророй и ментоном провести не удалось вследствие стерических затруднений. Установлено, что [5+1]-циклоконденсация исходных анилинов с гетероцикланонами (1-R-пиперидоном-4, дигидротиофен-3(2H)-оном, дигидро-2Н-пиран-4(3Н)-оном, дигидро-2H-тиопиран3(4H)-оном) протекает без особенностей с образованием соответствующих 2'-арил-6'H-спиро[1,2,4] триазоло[1,5-c]хиназолинов. Также без особенностей протекает реакция с 5-R-1H-индол-2,3-дионом (изатином) и его N-замещенными производными с образованием арил-2'-арил-6'H-спиро[(индол-3, 5'-[1,2, 4] триазоло[1,5-с]хиназолинов] с высокими выходами. Чистота синтезированных веществ была доказана методом LC-MS (APCI), их строение подтверждено комплексом фризико-химических методов, в частности ${ }^{1} \mathrm{H}$ u $^{13} \mathrm{C}$ ЯМР, ИК-, МС-(ЭУ)-спектрометрически и при помощи рентгеноструктурного анализа. Особенности ${ }^{1} \mathrm{H} \mathrm{u}{ }^{13} \mathrm{C}$ ЯМР-спектров синтезированных веществ были обговорены. Показано, что для синтезированных соединений характеристическими являются сигналы $\mathrm{NH}$-протонов в ${ }^{1} \mathrm{H}$ ямР спектре и С-5' в ${ }^{13} \mathrm{C}$ ЯМР-спектре.

Development and optimization of the existing synthetic methods of quinazoline and triazolo[c]quinazoline spiroderivatives are among important tasks of organic, bioorganic and medicinal chemistry. This group of compounds is of practical interest due to their high biological activity [1-3] and, at the same time, their original methods of synthesis. Reactions of [5+1]-cyclocondensation based on interactions of 1,5-binucleophiles with carbonyl compounds (cycloalkanones, 1-R-4-piperidone and others) can serve as an approach for construction of spiro compounds. Thus, the authors used 2-nitrobenzamide [4], 2-aminobenzamide and its derivatives $[2,5-8]$, or $1 H$-benzo[ $d][1,3]$ oxazine-2,4-dione [9] for the synthesis of 3'-R-1'-R-1' $H$ spiro[cycloalkane-1,2'-quinazoline]-4' $\left(3^{\prime} H\right)$-ones, oximes of 2-aminoacetophenone [3] for the synthesis of 4'-methyl-1',2'-dihydrospiro[cycloalkane-1,2'-quinazoline]-3-oxides, and 2-(aminomethyl)aniline $[10,11]$ for the synthesis of 3',4'-dihydro- 1 ' $H$-spiro[cyclohexane-1,2'-quinazoline]. However, only one publication was devoted to the synthesis of spiro[piperidine-4,5' $\left(6^{\prime} H\right)$-[1,2,4] triazolo[1,5-c]quinazolines], in which 2-(1H1,2,4-triazol-5-yl)aniline was used to form the systems mentioned [2]. Based on the above mentioned facts the aim of this work is to study the reactivity of [2-(3-aryl-1H-1,2,4-triazol-5-yl)phenyl]amines that are insufficiently studied as 1,5-binucleophiles in [5+1]-cyclocondensation reactions with cycloalkanones, heterocyclonones, isatines, and it can be one of the synthetic approaches for formation of the unstudied 2'-aryl-6' $H$-spiro[1,2,4] triazolo[1,5-c] quinazolines.

\section{Results and Discussion}

The starting [2-(3-aryl-1H-1,2,4-triazol-5-yl)phenyl] amines (1a-g) were synthesized according to the known method [12], namely nucleophilic cleavage of the pyrimidine ring of the corresponding 2 -aryl-[1,2,4] triazolo[1,5-c]quinazolines. The reaction of 1a-g with cycloalkanones (cyclopentanone, cyclohexanone) allowed to obtain products of binucleophilic addition, namely spiro derivatives $\mathbf{2 a - h}$ (Scheme). Furthermore, amine 1a readily reacts with a conformationally rigid bicyclo[2.2.1] heptan-2-one with formation of compound $\mathbf{2} \mathbf{i}$, whereas the reaction of amine $\mathbf{1 a}$ with camphor and menthone has failed due to the steric hindrance.

To study the reactivity of cycloalkanone with a heteroatom amines 1a-g were treated with heterocyclonones (1-R-piperidone-4, dihydrothiophene-3(2H)-one, dihydro- $2 H$-pyran- $4(3 H)$-one, dihydro- $2 H$-thiopyran$3(4 H)$-one). It was found that [5+1]-cyclocondensation of amines 1a-g with the corresponding electrophiles proceeded without peculiarities with formation of the corresponding 2'-aryl-6' $H$-spiro[1,2,4]triazolo[1,5-c]quinazolines (2d-h, Scheme). The [5+1]cyclocondensation of amines $\mathbf{1 a - g}$ with 5-R-1H-indole-2,3-dione (isatine) and its $\mathrm{N}$-substituted derivatives also proceeded without any peculiarities forming

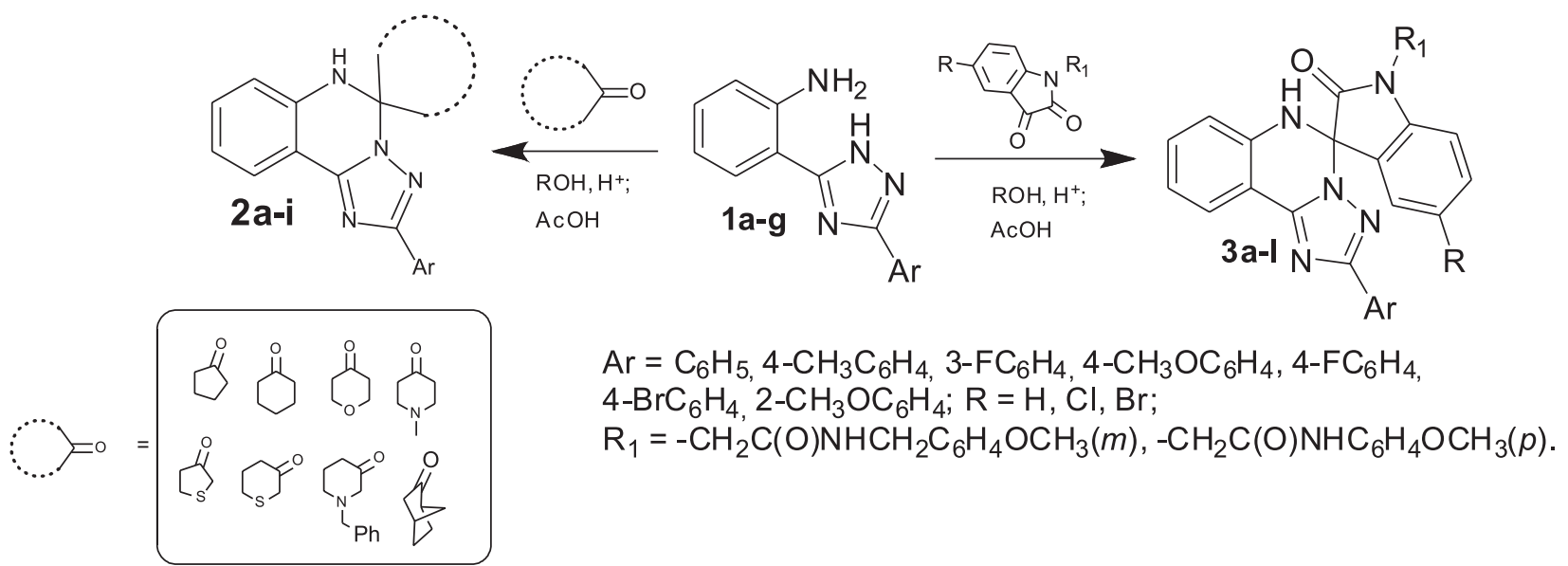

Scheme. The main approaches to the synthesis of 2'-aryl-6' $H$-spiro[1,2,4]triazolo[1,5-c]quinazolines. 
aryl-2'-aryl-6' $H$-spiro[(indol-3,5'-[1,2,4]triazolo[1,5-c] quinazolines] (3a-1, Scheme) with high yields. It was found that abovementioned reactions could be carried out in other organic solvents miscible with water and indifferent to the starting material that could significantly improve the yield and purity of the target compounds.

The purity of the compounds synthesized was proven by LC-MS (APCI) method, their structures were confirmed by the complex of physicochemical methods, including ${ }^{1} \mathrm{H}$ and ${ }^{13} \mathrm{C}$ NMR, IR-, MS-(EI)-spectrometry.

Aromatic protons of the triazolo[1,5-c]quinazoline cycle in the ${ }^{1} \mathrm{H}$ NMR-spectra of the compounds synthesized formed the characteristic ABCD system, which implements via two doublets (H-7, H-9) and two triplets $(\mathrm{H}-8, \mathrm{H}-10)$ with the corresponding chemical shifts. The characteristic signal of NH-group (6-positions of the dihydroquinazoline cycle) for compounds $\mathbf{2 a - h}, \mathbf{2} \mathbf{i}$ was observed in the spectra as a singlet at 7.26-6.83 ppm, and its chemical shift was determined by the size of a heteroatom in the spirocycle. It is likely that the most deshielded proton was the proton of the $\mathrm{NH}$ group of compound $\mathbf{2} \mathbf{f}$ with the thiophene ring. It was observed that the protons of the $\mathrm{NH}$-group (position 6) of compounds $\mathbf{3 a - l}$ were even more deshielded apparently due to the donoracceptor interaction with oxygen of the indole moiety and were recorded as singlets at 7.77-7.70 ppm. The other substituents in positions 2 ' and $5^{\prime}$ in the triazoloquinazoline system had "classical" signals of protons with the typical characteristic chemical shifts and multiplicity [13].

The ${ }^{13} \mathrm{C}$ NMR-spectra of compounds $2 \mathrm{a}, \mathbf{2 c}, \mathbf{2 i}$ and $3 \mathbf{a}$ additionally proved their structure. It is important that the characteristic signals of $\mathrm{sp}^{3}$-hybridized Carbon in positions $1,5^{\prime}$ were considerably deshielded and were observed at 82.93, 74.28, 81.38 and $75.51 \mathrm{ppm}$.

A characteristic feature of compound $\mathbf{2 c}$ in the EI-MS-spectrum was a fairly high intensity peak $[\mathrm{M}]^{+\bullet}$ $(\mathrm{m} / \mathrm{z} 316,72.9 \%)$ and two parallel fragmentation of the molecular ion. The ions $[\mathrm{M}-\mathrm{H}]^{+}(\mathrm{m} / \mathrm{z} 315,60.5 \%)$, $\left[\mathrm{M}-\mathrm{C}_{3} \mathrm{H}_{6}\right]^{+}(\mathrm{m} / \mathrm{z} 274,28.0 \%),\left[\mathrm{M}-\left(\mathrm{C}_{4} \mathrm{H}_{6}+\mathrm{H}\right)\right]^{+} \cdot(\mathrm{m} / \mathrm{z} 273$, $100.0 \%),\left[\mathrm{M}-\mathrm{C}_{4} \mathrm{H}_{8}\right]^{+}(\mathrm{m} / \mathrm{z} 260,5.6 \%),\left[\mathrm{M}-\left(\mathrm{C}_{4} \mathrm{H}_{8}+\mathrm{H}\right)\right]^{+}$ $(\mathrm{m} / \mathrm{z} 259,8,5 \%)$ formed were the most intense in the spectrum and characterized the main way of $[\mathrm{M}]^{+}$ fragmentation that passed through the cyclohexane fragment of the molecule. An alternative degradation of the molecule was associated with disruption of bonds $\mathrm{N}(1)-\mathrm{C}(2)$ and $\mathrm{N}(3)-\mathrm{N}(4)$ of the triazole ring and formation of fragmented ions $\left[\mathrm{M}-\mathrm{C}_{6} \mathrm{H}_{5} \mathrm{CN}\right]^{+}$ $(\mathrm{m} / \mathrm{z} 214,7,1 \%),\left[\mathrm{M}-\left(\mathrm{C}_{6} \mathrm{H}_{5} \mathrm{CN}+\mathrm{H}\right)\right]^{+\bullet}(\mathrm{m} / \mathrm{z} 213,21.8 \%)$. In addition, the EI-MS spectrum of compound $3 \mathbf{c}$ was characterized by the low-intensity molecular ion $[\mathrm{M}]^{+\bullet}$ $(\mathrm{m} / \mathrm{z} 395,2.3 \%)$, for which the first phase was characterized by release of $\mathrm{CO}$ and $\mathrm{H}$ particles with formation of ions $[\mathrm{M}-\mathrm{CO}]^{+}(\mathrm{m} / \mathrm{z} 368,7.4 \%)$, and $[\mathrm{M}-(\mathrm{CO}+\mathrm{H})]^{+}$. $(\mathrm{m} / \mathrm{z} 367,19.9 \%)$. Further fragmentation of $[\mathrm{M}-(\mathrm{CO}+\mathrm{H})]^{+\bullet}$ was associated with the alternative disruption of bonds $\mathrm{N}(1)-\mathrm{C}(2)$ and $\mathrm{N}(3)-\mathrm{N}(4), \mathrm{C}(10 \mathrm{~b})-\mathrm{N}(1)$ and $\mathrm{N}(3)-\mathrm{N}(4)$ of the triazole ring and resulted in formation of ions with $m / z 234$ (7.6\%) and 221 (15.3\%). It should be noted that the direction of fragmentation significantly differed from the 2 - $\mathrm{R}-[1,2,4]$ triazolo[1,5-c] quinazoline systems previously described [14]. In the systems mentioned fragmention of $[\mathrm{M}]^{+\bullet}$ was carried out by the cleavage of $\mathrm{C}(10 \mathrm{~b})-\mathrm{N}(1)$ and $\mathrm{N}(3)-\mathrm{N}(4)$ with formation of the amidine moiety and the fragmentary ion with the mass corresponding to quinazoline (m/z 129).

The IR-spectra of $\mathbf{2 a - i}$, in contrast to the starting amines 1a-g [12], had characteristic vibrations of the secondary $v_{\mathrm{NHR}_{2}}$-group as a single band in the region of 3396-3200 cm-1 $\mathrm{c}_{\mathrm{NH}}-1618-1589 \mathrm{~cm}^{-1}$ and low-intensity vibrations of $\mathrm{v}_{\mathrm{NHR}_{2}}$-group at 1650-1550 $\mathrm{cm}^{-1}$. The IR-spectra of compounds 3a-j had characteristic band vibrations of the associated form of $v_{\mathrm{NH}}$-lactams in the region of 3178-3016 $\mathrm{cm}^{-1}$ and vibrations of the $v_{\text {co }}$ group at $1748-1650 \mathrm{~cm}^{-1}$. In addition, the substituted indoles (3k and $\mathbf{3 l}$ ) had wider and more expressed intensity of stretching vibrations, indicating the presence of the primary amide group.

Additionally, the structure of compound 2a was determined by the X-ray diffraction study (Fig.). The tricyclic fragment was planar within 0.03 A. The spiro-joined tricycle and the pentane ring were orthogonal where the angle between the planar fragment of the cyclopentane ring was formed by C8, C16, C19 atoms, and the planar tricyclic fragment was $92^{\circ}$. The pentane ring was disordered over two positions (A and $B$ ) where the ratio $A: B$ is about $65: 35 \%$. Deviations of the C17 and C18 atoms from the mean plane of the remaining atoms of this ring were $0.45 \AA$ and $-0.24 \AA$, respectively, in conformer $A$ and $-0.20 \AA$ and $0.62 \mathrm{a}$, respectively, in conformer B. The $\mathrm{N} 2$ atom had a planar configuration, the sum of the bond angles centered at about $360^{\circ}$. The phenyl substituent was coplanar to the tricyclic fragment plane (the N4-C9-C10-C11 torsion angle iwa $\left.-0.5(2)^{\circ}\right)$, it was stabilized by the presence of H11...N4 (2.59 $)$ and H15...N1 (2.60 ̊) attractive interactions (the van der Waals radii sum $[15]$ is $2.67 \AA$ ). In the crystal phase molecules 2 a were bonded by the N2-H...N1' $(0.5+\mathrm{x}$, $0.5-\mathrm{y}, 0.5+\mathrm{z})$ intermolecular hydrogen bond $(\mathrm{H} \ldots \mathrm{N}$ $2.04 \AA \mathrm{N}-\mathrm{H}$.... $177^{\circ}$ ).

\section{Experimental Part}

Melting points were determined in open capillary tubes and were uncorrected. The elemental analyses (C, H, N, S) were performed using an ELEMENTAR vario EL Cube analyzer (USA). Analyses were indicated by the symbols of the elements or functions within $\pm 0.3 \%$ of the theoretical values. The IR-spectra (4000-600 $\mathrm{cm}^{-1}$ ) were recorded on a Bruker ALPHA 


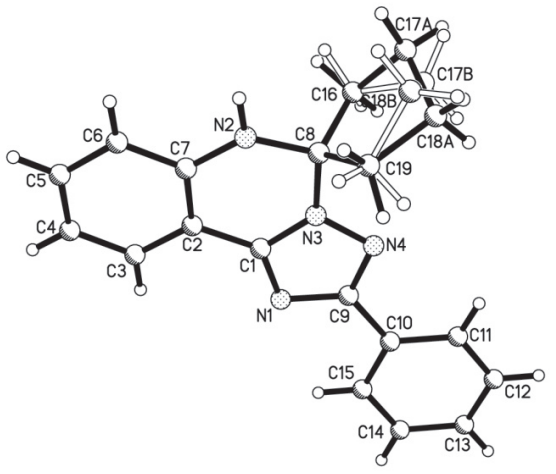

Fig. The molecular structure of compound $\mathbf{2 a}$ according to X-ray diffraction data.

FT-IR spectrometer (Bruker Bioscience, Germany) using a module for measuring attenuated total reflection (ATR). The ${ }^{1} \mathrm{H}$ NMR-spectra (400 MHz) and ${ }^{13} \mathrm{C}$ NMR-spectra $(100 \mathrm{MHz})$ were recorded on a VarianMercury 400 (Varian Inc., Palo Alto, CA, USA) spectrometer with TMS as an internal standard in DMSO- $\mathrm{d}_{6}$ solution. LC-MS were recorded using the chromatography/mass spectrometric system consisting of an "Agilent 1100 Series" high performance liquid chromatograph (Agilent, Palo Alto, CA, USA) equipped with an "Agilent LC/MSD SL" diode-matrix and mass-selective detector (atmospheric pressure chemical ionization - APCI). The electron impact mass spectra (EIMS) were recorded on a Varian $1200 \mathrm{~L}$ instrument at $70 \mathrm{eV}$ (Varian, USA).

Compounds 1a-g were obtained according to the synthetic protocols described [12], 5-R-1H-indol-2,3diones (isatines) and $\mathrm{N}$-substituted - by synthetic protocols [16]. The other starting reagents and solvents were obtained from commercially available sources and were used without further purification.

The general method for the synthesis of 2'-aryl$6^{\prime} \mathrm{H}$-spiro[1,2,4]triazolo[1,5-c]quinazolines (2a-i). To the solution of $10 \mathrm{mmol}$ of [2-(3-aryl- $1 \mathrm{H}-1,2,4-$ triazolo-5-yl)phenyl]amines (1a-g) in $10 \mathrm{ml}$ of propan-2-ol and 2 drops of conc. sulphuric acid (or $10 \mathrm{ml}$ of glacial acetic acid) add $10 \mathrm{mmol}$ of the corresponding cycloalkanone. Reflux the mixture for $6 \mathrm{~h}$, cool, pour into $10 \%$ solution of sodium acetate. Filter the precipitate formed and dry. If necessary, crystallize it with methanol.

2'-Phenyl-6'H-spiro[cyclopentane-1,5'-[1,2,4] triazolo[1,5-c]quinazoline] (2a). Yield - 92.7\%. M. p. $-180-181^{\circ} \mathrm{C} ; \mathrm{IR}, \mathrm{v}, \mathrm{cm}^{-1}: 3200,3105,3034,2955$, $2870,1728,1621,1593,1519,1485,1469,1441,1409$, $1349,1330,1278,1265,1200,1177,1152,1125,1106$, $1069,1035,982,956,936,913,856,782,765,743$, 718, 683, 668, 631, 617; ${ }^{1} \mathrm{H}$ NMR, $\delta$, ppm. (J, Hz): 8.09 $(\mathrm{d}, J=7.2 \mathrm{~Hz}, 1 \mathrm{H}, \mathrm{H}-2,6 \mathrm{Ph}), 7.74(\mathrm{~d}, J=7.4 \mathrm{~Hz}, 1 \mathrm{H}$, $\mathrm{H}-10$ ), 7.52-7.29 (m, 3H, H-3,4,5 Ph), 7.19 (t, $J=7.5 \mathrm{~Hz}$, $1 \mathrm{H}, \mathrm{H}-8), 6.97$ (s, $1 \mathrm{H}, \mathrm{NH}$ quin.), 6.84 (d, $J=8.0 \mathrm{~Hz}$, $1 \mathrm{H}, \mathrm{H}-7), 6.78(\mathrm{t}, J=7.3 \mathrm{~Hz}, 1 \mathrm{H}, \mathrm{H}-9), 2.54-2.36$ (m, 4H, H-2,2, 5,5 cyclopentane), 2.13-1.85 (m, 4H, H-3,3, 4,4 cyclopentane; ${ }^{13} \mathrm{C}$ NMR, $\delta$, ppm: 160.77 (C-2'), 149.64 (C-6a'), 142.96 (C-10b'), 131.72, 131.11, 129.23, $128.77,125.97,124.04,118.38,114.98,110.54$ (C-10a'), 82.93 (C-1,5'), 38.95 (C-2,5), 23.57 (C-3,4); LC-MS, $m / z=303.2[\mathrm{M}+1]$; Anal. Calcd for $\mathrm{C}_{19} \mathrm{H}_{18} \mathrm{~N}_{4}$ : C, 75.47; H, 6.00; N, 18.53; Found: C, 75.43; H, 5.98; N, 18.49.

2'-(3-Fluorophenyl)-6' H-spiro[cyclopentane-1,5'-[1,2,4]triazolo[1,5-c]quinazoline] (2b). Yield - 52.1\%. M. p. $-140-142^{\circ} \mathrm{C}$; IR, v, $\mathrm{cm}^{-1}$ : 3234, 2954, 2917, 2849, 1630, 1592, 1561, 1530, 1514, $1480,1452,1413,1387,1366,1330,1308,1264$, $1210,1165,1154,1114,1095,1068,1035,983$, $963,900,877,779,743,720,678,636,616 ;{ }^{1} \mathrm{H}$ NMR, $\delta$, ppm. $(J, \mathrm{~Hz}): 7.91$ (d, $J=7.6 \mathrm{~Hz}, 1 \mathrm{H}, \mathrm{H}-10), 7.83-$ 7.69 (m, 2H, H-2,6 3-FPh), 7.43 (dd, $J=13.8,7.7 \mathrm{~Hz}$, $1 \mathrm{H}, \mathrm{H}-53-\mathrm{FPh}), 7.19(\mathrm{t}, J=7.6 \mathrm{~Hz}, 1 \mathrm{H}, \mathrm{H}-8), 7.10(\mathrm{t}, J$ $=8.0 \mathrm{~Hz}, 1 \mathrm{H}, \mathrm{H}-4 \mathrm{3}-\mathrm{FPh}), 7.01(\mathrm{~s}, 1 \mathrm{H}, \mathrm{NH}$ quin.), 6.84 (d, $J=8.0 \mathrm{~Hz}, 1 \mathrm{H}, \mathrm{H}-7), 6.78$ (t, $J=7.4 \mathrm{~Hz}, 1 \mathrm{H}, \mathrm{H}-9$ ), 2.56-2.34 (m, 4H, H-2,2, 5,5 cyclopentane), 2.141.85 (m, 4H, H-3,3, 4,4 cyclopentane); LC-MS, $m / z=$ 321.2 [M+1]; Anal. Calcd for $\mathrm{C}_{19} \mathrm{H}_{17} \mathrm{FN}_{4}$ : C, 71.23; $\mathrm{H}$, 5.35; N, 17.49; Found: C, 71.24; H, 5.39; N, 17.52.

2'-Phenyl-6'H-spiro[cyclohexane-1,5'-[1,2,4]triazolo[1,5-c]quinazoline] (2c). Yield - 98.6\%. M. p. $141-143^{\circ} \mathrm{C}$; IR v, $\mathrm{cm}^{-1}: 3378,3275,3255,3227,2960$, $2930,2855,1728,1620,1586,1538,1514,1496,1480$, $1468,1441,1408,1343,1317,1289,1268,1242,1211$, $1168,1156,1145,1126,1110,1072,1056,1043,1030$, $1003,984,972,943,919,908,857,785,753,729,720$, $698,688,668,659,632 ;{ }^{1} \mathrm{H}$ NMR, $\delta$, ppm. $(J, \mathrm{~Hz})$ 8.09 (d, $J=7.1 \mathrm{~Hz}, 2 \mathrm{H}, \mathrm{H}-2,6 \mathrm{Ph}), 7.75$ (d, $J=7.4 \mathrm{~Hz}$, $1 \mathrm{H}, \mathrm{H}-10), 7.47-7.38$ (m, 2H, H-3,5 Ph), 7.36 (d, $J=$ $6.0 \mathrm{~Hz}, 1 \mathrm{H}, \mathrm{H}-4 \mathrm{Ph}), 7.20$ (t, J = 7.5 Hz, 1H, H-8), 7.02 (d, $J=7.9 \mathrm{~Hz}, 1 \mathrm{H}, \mathrm{H}-7), 6.87-6.72(\mathrm{~m}, 2 \mathrm{H}, \mathrm{NH}$ quin., $\mathrm{H}-9$ ), 2.17 (d, $J=10.7 \mathrm{~Hz}, 1 \mathrm{H}), 2.03(\mathrm{~d}, J=12.3 \mathrm{~Hz}$, $1 \mathrm{H}), 1.88-1.64(\mathrm{~m}, 3 \mathrm{H}), 1.39(\mathrm{~d}, J=8.2 \mathrm{~Hz}, 1 \mathrm{H}) ;{ }^{13} \mathrm{C} \mathrm{NMR}$, $\delta$, ppm: 160.59 (C-2'), 149.38 (C-6a'), 142.25 (10b'), 131.80, 131.16, 129.21, 128.77, 125.93, 123.97, 118.48, 115.60, 110.69 (10a'), 74.28 (C-1,5'), 35.35, 24.40, 20.96; EI-MS: $m / z=316$ (72.9. $\left.\mathrm{M}^{+}\right), 315$ (60.5), 274 (28.0), 273 (100.0), 260 (5.6), 259 (8.5), 258 (5.1), 248 (6.5), 214 (7.1), 213 (21.8), 184 (6.7), 155 (15.2), 154 (9.3), 149 (7.6), 129 (6.6), 127 (10.1), 123 (5.2), 119 (5.1), 118 (7.2), 103 (11.6), 94 (9.9), 90 (10.9), 77 (8.3), 57 (11.7), 56 (5.1), 55 (19.6), 54 (5.8); LC-MS, $m / z=317.2[\mathrm{M}+1]$; Anal. Calcd for $\mathrm{C}_{20} \mathrm{H}_{20} \mathrm{~N}_{4}$ : C, 75.92; H, 6.37; N, 17.71; Found: C, 75.94; H, 6.39; N, 17.75.

2'-Phenyl-2,3,5,6-tetrahydro-6'H-spiro[pyran4,5'-[1,2,4] triazolo[1,5-c]quinazoline] (2d). Yield 57.8\%. M. p. $-150-151^{\circ} \mathrm{C} ; \mathrm{IR}, \mathrm{v}, \mathrm{cm}^{-1}: 3313,2949,2920$, $2848,1623,1559,1517,1507,1478,1465,1440,1386$, $1360,1342,1320,1300,1280,1208,1155,1124,1097$, $1069,1026,1010,994,921,858,829,774,746,723$, $692,675,634,619 ;{ }^{1} \mathrm{H}$ NMR, $\delta$, ppm. $(J, \mathrm{~Hz}): 8.09$ (d, $J$ $=7.2 \mathrm{~Hz}, 1 \mathrm{H}, \mathrm{H}-2,6 \mathrm{Ph}), 7.76(\mathrm{~d}, J=7.4 \mathrm{~Hz}, 1 \mathrm{H}, \mathrm{H}-10)$, 7.47-7.31 (m, 3H, H-3,4,5 Ph), 7.21 (t, J= 7.3 Hz, 1H, 
H-8), 7.09 (s, 1H, NH quin.), 7.00 (d, $J=8.0 \mathrm{~Hz}, 1 \mathrm{H}$, $\mathrm{H}-7), 6.82$ (t, $J=7.3 \mathrm{~Hz}, 1 \mathrm{H}, \mathrm{H}-9), 4.03-3.81(\mathrm{~m}, 4 \mathrm{H}$, $\left.\mathrm{O}\left(\mathrm{CH}_{2}\right)_{2}\right), 2.50-2.35$ (m, 4H. C $\left.\left(\mathrm{CH}_{2}\right)_{2}\right)$; LC-MS, $m / z=$ $319.2[\mathrm{M}+1]$; Anal. Calcd for $\mathrm{C}_{19} \mathrm{H}_{18} \mathrm{~N}_{4} \mathrm{O}$ : C, 71.68; $\mathrm{H}$, 5.70; N, 17.60; Found: C, 71.69; H, 5.73; N, 17.61.

1-Methyl-2'-phenyl-6'H-spiro[piperidine4,5'-[1,2,4]triazolo[1,5-c]quinazoline] (2e). Yield - 83.6\%. M. p. $-212-214^{\circ} \mathrm{C}$; IR, v, cm-1: 3396, 3341, 3226, 3061, 3016, 1621, 1596, 1546, 1517, 1479, 1436, 1406, 1339, 1304, 1254, 1149, 1122, 1111, 1069, 1048, 1019, 998, 973, 927, 864, 786, 768, 751, 734, 719, 688, 673, 664, 613; ${ }^{1} \mathrm{H}$ NMR, $\delta$, ppm. $(J, \mathrm{~Hz}): 8.09$ (d, $J$ $=7.0 \mathrm{~Hz}, 2 \mathrm{H}, \mathrm{H}-2,6 \mathrm{Ph}), 7.77$ (d, $J=7.4 \mathrm{~Hz}, 1 \mathrm{H}, \mathrm{H}-10)$, 7.51-7.32 (m, 3H, H-3,4,5 Ph), 7.24 (t, J = 7.3 Hz, 1H, H-8), 7.16 (s, 1H, NH quin.), 7.07 (d, $J=7.7 \mathrm{~Hz}, 1 \mathrm{H}$, $\mathrm{H}-7), 6.83$ (t, $J=7.1 \mathrm{~Hz}, 1 \mathrm{H}, \mathrm{H}-9), 3.34-3.06(\mathrm{~m}, 4 \mathrm{H}$, $\mathrm{H}-2,6$ piperidine), $2.67\left(\mathrm{~s}, 3 \mathrm{H},-\mathrm{CH}_{3}\right), 2.65-2.43(\mathrm{~m}$, $4 \mathrm{H}, \mathrm{H}-3,5$ piperidine); Anal. Calcd for $\mathrm{C}_{20} \mathrm{H}_{21} \mathrm{~N}_{5}$ : C, 72.48; H, 6.39; N, 21.13; Found: C, 72.48; H, 6.41; N, 21.12 .

2'-Phenyl-4,5-dihydro-6'H-spiro[thiophene3,5'-[1,2,4]triazolo[1,5-c]quinazoline] (2f). Yield - 47.8\%. M. p. $-195-197^{\circ} \mathrm{C}$; IR, v, $\mathrm{cm}^{-1}$ : 2922, 1742 , $1631,1610,1562,1532,1519,1479,1463,1441,1427$, $1385,1360,1348,1322,1301,1275,1172,1153,1112$, 1069, 1026, 987, 959, 928, 863, 792, 774, 737, 723, 693, 678, 662, 634, 616; ${ }^{1} \mathrm{H}$ NMR, $\delta$, ppm. (U, Hz): 8.09 (d, $J=7.2 \mathrm{~Hz}, 2 \mathrm{H}, \mathrm{H}-2,6 \mathrm{Ph}$ ), 7.77 (d, $J=7.5 \mathrm{~Hz}, 2 \mathrm{H}$, H-10), 7.45-7.32 (m, 3H, H-3,4,5 Ph), 7.26 (s, 1H, NH quin.), $7.22(\mathrm{t}, J=7.3 \mathrm{~Hz}, 1 \mathrm{H}, \mathrm{H}-8), 6.97(\mathrm{~d}, J=8.1 \mathrm{~Hz}$, 1H, H-7), 6.81 (d, J = 7.0 Hz, 1H, H-9), 3.49 (d, J= 11.3 $\mathrm{Hz}, 1 \mathrm{H},-\underline{\mathrm{H}}_{2}-\mathrm{S}-$ ), 3.24-3.06 (m, 3H, $\left.-\mathrm{CH}_{2}-\mathrm{S}-,-\mathrm{CCH}_{2}\right)$ ), 2.94-2.64 (m, 1H, -SC $\underline{H}_{2}{ }^{-}$), 2.59-2.51 (m, 1H, -SC $\underline{H}_{2}-$ ); Anal. Calcd for $\mathrm{C}_{18} \mathrm{H}_{16} \mathrm{~N}_{4} \mathrm{~S}: \mathrm{C}, 67.47 ; \mathrm{H}, 5.03 ; \mathrm{N}, 17.49$; Found: C, 67.45; H, 5.01; N, 17.46.

2'-Phenyl-2,4,5,6-tetrahydro-6'H-spiro[thiopyran-3,5'-[1,2,4]triazolo[1,5-c]quinazoline] (2g). Yield - 98.7\%. M. p. $-164-166^{\circ} \mathrm{C}$; IR, v, cm-1 3371 , 2920, 2851, 1732, 1620, 1591, 1539, 1519, 1493, 1478, $1441,1429,1386,1360,1343,1320,1300,1281,1254$, $1173,1158,1125,1113,1093,1069,1045,1026,972$, 922, 898, 863, 789, 774, 745, 723, 692, 664, 643, 614; ${ }^{1} \mathrm{H}$ NMR, $\delta$, ppm. $(J, \mathrm{~Hz}): 8.08$ (d, $\left.J=7.2 \mathrm{~Hz}, 2 \mathrm{H}, \mathrm{H}-2,6 \mathrm{Ph}\right)$, 7.76 (d, J = 7.5 Hz, 1H, H-10), 7.48-7.31 (m, 3H, H-3,4,5 $\mathrm{Ph}), 7.22$ (t, $J=7.3 \mathrm{~Hz}, 1 \mathrm{H}, \mathrm{H}-8), 7.14(\mathrm{~d}, J=8.0 \mathrm{~Hz}$, 1H, H-7), 7.01 (s, 1H, NH quin.), 6.82 (t, $J=7.2 \mathrm{~Hz}$, $1 \mathrm{H}, \mathrm{H}-9), 3.33,3.01$ (d, $J=13.2,11.1 \mathrm{~Hz}, 2 \mathrm{H},-\underline{\mathrm{H}}_{2} \mathrm{~S}-$ ), 2.78, 2.68 (d, $J=13.1,11.1 \mathrm{~Hz}, 2 \mathrm{H},-\mathrm{SC}_{\mathrm{H}_{2}}-$ ), 2.46-2.23 (m, 2H, -CC $\underline{H}_{2}^{-}$), $2.24-2.11$ (m, 2H, $-\mathrm{SCH}_{2} \underline{\mathrm{CH}}_{2}$ ); LC-MS, $m / z=335.1[\mathrm{M}+1]$; Anal. Calcd for $\mathrm{C}_{19} \mathrm{H}_{18} \mathrm{~N}_{4} \mathrm{~S}: \mathrm{C}, 68.23$; H, 5.42; N, 16.75; S, 9.59; Found:C, 68.26; H, 5.45; N, 16.76 .

1-Benzyl-2' -phenyl-6'H-spiro[piperidine3,5'-[1,2,4]triazolo[1,5-c]quinazoline] (2h). Yield - 74.7\%. M. p. $-295-297^{\circ} \mathrm{C}$; IR, v, cm${ }^{-1}: 1739,1621$, $1591,1517,1506,1472,1441,1407,1390,1342,1260$, $1193,1153,1107,1063,1027,991,973,947,927$,
$889,866,806,787,748,724,694,666,636,622 ;{ }^{1} \mathrm{H}$ NMR, $\delta$, ppm. $(J, H z): 8.08$ (d, 2H, H-2,6), $7.74(\mathrm{~d}, J=$ $7.5 \mathrm{~Hz}, 1 \mathrm{H}, \mathrm{H}-10$ ), 7.50 (t, $J=7.5 \mathrm{~Hz}, 1 \mathrm{H}, \mathrm{H}-8$ ), 7.457.13 (m, 8H, H-3,4,5 Ph, H-2,3,4,5,6-CH $\left.{ }_{2} \mathrm{Ph}\right), 7.01$ (d, $J=8.0 \mathrm{~Hz}, 1 \mathrm{H}, \mathrm{H}-7), 6.87(\mathrm{~s}, 1 \mathrm{H}, \mathrm{NH}$ quin.), 6.79 (t, $J$ $=7.3 \mathrm{~Hz}, 1 \mathrm{H}, \mathrm{H}-9), 3.59,3.03\left(\mathrm{~s}, 2 \mathrm{H},-\mathrm{CH}_{2}\right), 2.79(\mathrm{~m}$, $2 \mathrm{H}, \mathrm{H}-2$ piperidine), 2.59 ( $\mathrm{m}, 2 \mathrm{H}, \mathrm{H}-6$ piperidine), $2.00(\mathrm{~d}, J=12.4 \mathrm{~Hz}, 2 \mathrm{H}, \mathrm{H}-4$ piperidine), $1.89(\mathrm{~m}, 2 \mathrm{H}$, $\mathrm{H}-5$ piperidine); Anal. Calcd for $\mathrm{C}_{26} \mathrm{H}_{25} \mathrm{~N}_{5}$ : C, 76.63; H, 6.18; N, 17.19; Found: C, 76.67; H, 6.21; N, 17.21.

2'-Phenyl-6'H-spiro[bicyclo[2.2.1] heptane2,5'-[1,2,4]triazolo[1,5-c]quinazoline] (2i). Yield - 67.1\%. M. p. $-237-239^{\circ} \mathrm{C}$; IR, v, $\mathrm{cm}^{-1}$ : 2959, 2928, 2872, 1723, 1622, 1591, 1540, 1518, 1505, 1495, 1443, 1384, 1327, 1276, 1218, 1182, 1156, 1110, 1097, 1073, $1013,987,950,929,892,857,824,775,746,723,710$, $688,669,654,641,627 ;{ }^{1} \mathrm{H}$ NMR, $\delta$, ppm. (J, Hz): 8.16 (m, 2H, H-2,6 Ph), 7.77 (d, J = 7.4 Hz, 1H, H-10), 7.497.31 (m, 3H, H-3,4,5 Ph), $7.22(\mathrm{dd}, J=13.3,6.6 \mathrm{~Hz}$ $1 \mathrm{H}, \mathrm{H}-8), 6.94$ (dd, $J=17.0,8.0 \mathrm{~Hz}, 1 \mathrm{H}, \mathrm{H}-7), 6.83(\mathrm{~m}$, 2H, NH quin., H-9), 3.20, 2.23 (d, J=12.1 Hz, 1H, H-4 bicyclo[2.2.1] heptane), 2.82, 2.00 (d, $J=13.1 \mathrm{~Hz}, 1 \mathrm{H}$, $\mathrm{H}-1$ bicyclo[2.2.1] heptane), 2.43, 1.91 (m, 2H, H-2 bicyclo[2.2.1] heptane), 1.66, 1.23 (m, 4H, H-5,6 bicyclo [2.2.1]heptane), 1.36, 1.11 (m, 2H, H-7 bicyclo[2.2.1] heptane); ${ }^{13} \mathrm{C}$ NMR, $\delta$, ppm: 159.41 (C-2'), 150.06 (C6a'), 143.24 (C-10b'), 131.97, 130.00, 128.81, 126.05, 124.24, 118.77, 115.38, 111.57 (C-10a'), 81.38 (C-2,5'), 47.14, 42.25, 36.91, 36.59, 27.73, 23.35; LC-MS, $m / z$ = $329.1[\mathrm{M}+1]$; Anal. Calcd for $\mathrm{C}_{21} \mathrm{H}_{21} \mathrm{ClN}_{4}$ : C, 69.13; H, 5.80; Cl, 9.72; N, 15.36; Found: C, 69.11; H, 5.77; $\mathrm{N}, 15.33$.

The general method for the synthesis of 2'-aryl6'H-spiro[(indole-3,5'-[1,2,4]triazolo[1,5-c]quinazolines] (3a-j) and $\mathrm{N}$-substituted derivatives (3k, 3l). To the solution of $10 \mathrm{mmol}$ of [2-(3-aryl- $1 \mathrm{H}-1,2,4-$ triazolo-5-yl)phenyl]amines (1a, 1c-g) in $10 \mathrm{ml}$ of propan-2-ol and 2 drops of conc. sulphuric acid (or $10 \mathrm{ml}$ of glacial acetic acid) add $10 \mathrm{mmol}$ of the corresponding 5-R-1H-indol-2,3-diones (isatines) or its $\mathrm{N}$-substituent. Boil the reaction mixture for $6 \mathrm{~h}$, cool, pour into $10 \%$ solution of sodium acetate, filter the precipitate formed and dry. If necessary, crystallize the precipitate with methanol.

2'-Phenyl-6'H-spiro[indole-3,5'-[1,2,4]triazolo[1,5-c]quinazolin]-2(1H)-one (3a). Yield - 98.3\%. M. p. $-242-244^{\circ} \mathrm{C}$; IR, $v, \mathrm{~cm}^{-1}: 3169,3098,3032,2973$, $2835,2613,1734,1700,1621,1593,1573,1544,1519$, $1472,1443,1400,1346,1319,1254,1187,1153,1115$, $1106,1097,1072,1038,961,923,878,863,850,778$, $744,725,708,688,650,624 ;{ }^{1} \mathrm{H}$ NMR, $\delta$, ppm. $(J, \mathrm{~Hz})$ : 10.55 (s, $1 \mathrm{H}, \mathrm{NH}$ indol), 8.00 (d, $J=7.0 \mathrm{~Hz}, 2 \mathrm{H}, \mathrm{H}-2,6$ $\mathrm{Ph}), 7.84(\mathrm{~d}, J=7.4 \mathrm{~Hz}, 1 \mathrm{H}, \mathrm{H}-10), 7.74(\mathrm{~s}, 1 \mathrm{H}, \mathrm{NH}$ quin.), 7.45-7.30 (m, 5H, H-3,4,5 Ph, H-4,6 indol), 7.23 $(\mathrm{t}, J=7.5 \mathrm{~Hz}, 1 \mathrm{H}, \mathrm{H}-8), 7.06(\mathrm{t}, J=7.2 \mathrm{~Hz}, 1 \mathrm{H}, \mathrm{H}-9)$, 6.99 (d, $J=7.5 \mathrm{~Hz}, 1 \mathrm{H}, \mathrm{H}-7), 6.91-6.76$ (m, 2H, H-5,7 indol); ${ }^{13} \mathrm{C}$ NMR, $\delta$, ppm: 173.00 (C-2), 162.00 (C-2'), 
151.44 (C-10b'), 142.53 (C-6a'), 142.48 (C-7a), 142.44, $132.14,131.85,130.27,129.66,129.62,128.83,127.32$, $126.14,125.87,124.08,123.00,118.68,114.43,110.91$, 109.36 (C-10a'), 75.51 (C-3,5'); LC-MS, $m / z=366.0$ $[\mathrm{M}+1]$; Anal. Calcd for $\mathrm{C}_{22} \mathrm{H}_{15} \mathrm{~N}_{5} \mathrm{O}: \mathrm{C}, 72.32 ;$ H. 4.14; N. 19.17; Found: C. 72.36; H. 4.17; N. 19.21.

2'-(4-Fluorophenyl)-6' H-spiro[indole-3,5' $[1,2,4]$ triazolo[1,5-c]quinazoline]-2(1H)-one (3b). Yield - 63.8\%. M. p. - 295-297 ${ }^{\circ}$; IR, v, cm-1 3164 , 3098, 2950, 2914, 1731, 1621, 1600, 1544, 1511, 1462, $1444,1415,1360,1315,1289,1260,1223,1197,1186$, $1148,1134,1107,1090,1012,980,964,940,913,900$, 844, 816, 784, 765, 748, 741, 722, 709, 684, 659, 624; ${ }^{1} \mathrm{H}$ NMR, $\delta$, ppm. $(J, \mathrm{~Hz}): 10.54$ (s, 1H, NH indol), 8.087.97 (m, 2H, H-2,6 Ph), 7.81 (d, $J=7.5 \mathrm{~Hz}, 1 \mathrm{H}, \mathrm{H}-10$ ), 7.71 (s, 1H, NH quin.), 7.43-7.32 (m, 2H, H-4,6 indol), 7.22 (t, $J=7.5 \mathrm{~Hz}, 1 \mathrm{H}, \mathrm{H}-8), 7.15-7.02$ (m, 2H, H-9, $\mathrm{H}-3,5 \mathrm{Ph}), 6.97$ (d, J = 7.7 Hz, 1H, H-7), 6.88-6.77 (m, 2H, H-5,7 indol); LC-MS, m/z = 384.1 [M + 1]; Anal. Calcd for $\mathrm{C}_{22} \mathrm{H}_{15} \mathrm{FN}_{5} \mathrm{O}$ : C, 68.92; H. 3.68; N. 18.27; Found: C. 68.96; H. 3.71; N. 18.29.

2'-(2-Methoxyphenyl)-6'H-spiro[indole-3,5' $[1,2,4]$ triazolo[1,5-c]quinazoline]-2(1H)-one (3c). Yield - 62.7\%. M. p. $-237-239^{\circ} \mathrm{C}$; IR, v, $\mathrm{cm}^{-1}: 3175$, 3100, 3069, 3026, 2951, 2830, 1733, 1620, 1603, 1559, $1515,1472,1456,1437,1428,1405,1318,1286,1269$, $1244,1199,1179,1155,1124,1106,1087,1044,1020$, 979, 965, 945, 848, 743, 718, 683, 664, 623; ${ }^{1} \mathrm{H}$ NMR, $\delta$, ppm. $(J, \mathrm{~Hz}): 10.52$ (s, $1 \mathrm{H}, \mathrm{NH}$ indol), 7.80 (d, $J=$ $7.5 \mathrm{~Hz}, 1 \mathrm{H}, \mathrm{H}-10$ ), 7.70 (s, 1H, NH quin.), 7.63 (d, $J=$ $7.2 \mathrm{~Hz}, 1 \mathrm{H}, \mathrm{H}-6 \mathrm{Ph}), 7.45-7.28$ (m, 3H, H-4,6 indol, $\mathrm{H}-4 \mathrm{Ph}), 7.21$ (t, $J=7.4 \mathrm{~Hz}, 1 \mathrm{H}, \mathrm{H}-8), 7.04$ (t, $J=7.4 \mathrm{~Hz}$, 1H, H-9), 7.03-6.90 (m, 3H, H-5,7 indol, H-7), 6.88$6.78(\mathrm{~m}, 2 \mathrm{H}, \mathrm{H}-3,5 \mathrm{Ph}), 3.76\left(\mathrm{~s}, 3 \mathrm{H},-\mathrm{OCH}_{3}\right)$; LC-MS, $\mathrm{m} / \mathrm{z}=396.1[\mathrm{M}+1]$; EI-MS: $m / z=395\left(2.3 . \mathrm{M}^{++}\right)$, 368 (7.4), 367 (19.9), 323 (6.4), 321 (5.1), 266 (8.9), 262 (6.5), 261 (5.8), 260 (11.5), 236 (12.3), 234 (7.6), 221 (15.3), 220 (11.7), 219 (5.3), 205 (7.8), 118 (9.3), 111 (9.0), 91 (9.5), 90 (8.6), 86 (30.7), 84 (100.0), 83 (17.8), 82 (11.7), 81 (9.0), 78 (6.3), 77 (11.1), 76 (5.5), 57 (8.7), 55 (10.7), 51 (85.0), 50 (22.6); Anal. Calcd for $\mathrm{C}_{23} \mathrm{H}_{17} \mathrm{~N}_{5} \mathrm{O}_{2}$ : C, 69.86; H. 4.33; N. 17.71; Found: C. 69.90; H. 4.37; N. 17.74.

5-Chloro-2' - phenyl-6'H-spiro[indole-3,5' $[1,2,4]$ triazolo[1,5-c]quinazoline]-2(1H)-one (3d). Yield $-85.7 \%$. M. p. > $300^{\circ} \mathrm{C}$; IR, v, cm $\mathrm{cm}^{-1}$ : 3176, 3141, 3100, 3016, 2938, 2858, 1733, 1681, 1621, 1594, 1545, $1518,1480,1441,1400,1345,1318,1282,1264,1197$, $1181,1151,1140,1107,1069,1027,1006,977,950$, 937, 922, 892, 874, 847, 821, 801, 787, 769, 743, 721, 686, 638; ${ }^{1} \mathrm{H}$ NMR, $\delta$, ppm. $(J, \mathrm{~Hz}): 10.67$ (s, $1 \mathrm{H}, \mathrm{NH}$ indol), 8.01 (d, $J=6.8 \mathrm{~Hz}, 2 \mathrm{H}, \mathrm{H}-2,6 \mathrm{Ph}), 7.84(\mathrm{~d}, J=$ $7.5 \mathrm{~Hz}, 1 \mathrm{H}, \mathrm{H}-10$ ), 7.75 (s, 1H, NH quin.), 7.45-7.29 (m, 5H, H-4,6 indol, H-3,4,5 Ph), $7.24(\mathrm{t}, J=7.4 \mathrm{~Hz}, 1 \mathrm{H}$, $\mathrm{H}-8), 6.99$ (d, $J=8.6 \mathrm{~Hz}, 1 \mathrm{H}, \mathrm{H}-7), 6.92-6.76(\mathrm{~m}, 2 \mathrm{H}$, $\mathrm{H}-9, \mathrm{H}-7$ indol); LC-MS, $\mathrm{m} / \mathrm{z}=400.0[\mathrm{M}+1]$; Anal.
Calcd for $\mathrm{C}_{22} \mathrm{H}_{14} \mathrm{ClN}_{5} \mathrm{O}$ : C, 66.09; H. 3.53; N. 17.52; Found: C. 66.06; H. 3.50; N. 17.50.

5-Chloro-2'-(4-fluorophenyl)-6'H-spiro[indole3,5'-[1,2,4]triazolo[1,5-c]quinazoline]-2(1H)one (3e). Yield - 99.9\%. M. p. $-280-282^{\circ} \mathrm{C}$; IR, v, $\mathrm{cm}^{-1}$ : 3173, 3131, 3105, 3018, 2945, 1732, 1713, 1621, 1603, $1547,1517,1480,1470,1446,1417,1359,1287,1264$, $1233,1222,1198,1184,1152,1127,1109,1092,1071$, $1047,1013,980,940,914,898,874,846,819,783$, 764, 748, 720, 708, 630, 614; ${ }^{1} \mathrm{H}$ NMR, $\delta$, ppm. (J, Hz): 10.69 (s, 1H, NH indol), 8.03 (t, $J=6.7 \mathrm{~Hz}, 1 \mathrm{H}, \mathrm{H}-2,6$ $\mathrm{Ph}), 7.82$ (d, $J=7.0 \mathrm{~Hz}, 1 \mathrm{H}, \mathrm{H}-10), 7.77$ (s, 1H, NH quin.), 7.38 (m, 2H, H-4,6 indol), 7.25 (t, $J=7.4 \mathrm{~Hz}$, 1H, H-8), 7.10 (t, J = 8.5 Hz, 2H, H-3,5 Ph), 6.98 (d, J = 8.7 Hz, 1H, H-7), 6.90-6.78 (m, 2H, H-9, H-7 indol); LC-MS, m/z = $418.0[\mathrm{M}+1]$; Anal. Calcd for $\mathrm{C}_{22} \mathrm{H}_{13} \mathrm{ClFN}_{5} \mathrm{O}$ : C, 63.24; H. 3.14; N. 16.76. Found: C. 63.27; H. 3.18; N. 16.79 .

5-Chloro-2'-(2-methoxyphenyl)-6'H-spiro[indole$3,5^{\prime}-[1,2,4]$ triazolo[1,5-c]quinazoline $]-2(1 \mathrm{H})$ one (3f). Yield $-45.4 \%$. M. p. $-247-249^{\circ} \mathrm{C}$; IR, v, $\mathrm{cm}^{-1}$ : $1726,1713,1681,1650,1613,1545,1536,1512,1477$, $1462,1454,1442,1400,1290,1275,1251,1181,1147$, $1109,1067,1048,1021,975,897,878,819,801,753$, 725, 689, 669, 660, 626, 613; ${ }^{1} \mathrm{H}$ NMR, $\delta$, ppm. (J, Hz): 10.66 (s, 1H, NH indol), 7.96 (d, $J=7.5 \mathrm{~Hz}, 1 \mathrm{H}, \mathrm{H}-10)$, 7.74 (s, 1H, NH quin.), 7.64 (d, J= 7.2 Hz, 1H, H-6 Ph), 7.45-7.28 (m, 3H, H-4,6 indol, H-4 Ph), 7.24 (t, $J=$ $7.4 \mathrm{~Hz}, 1 \mathrm{H}, \mathrm{H}-8$ ), 7.02 (t, $J=7.4 \mathrm{~Hz}, 1 \mathrm{H}, \mathrm{H}-9$ ), 7.036.90 (m, 3H, H-7 indol, H-7), 6.88-6.78 (m, 2H, H-3,5 $\mathrm{Ph}), 3.76\left(\mathrm{~s}, 3 \mathrm{H},-\mathrm{OCH}_{3}\right)$; LC-MS, $\mathrm{m} / \mathrm{z}=430.0[\mathrm{M}+1]$; Anal. Calcd for $\mathrm{C}_{23} \mathrm{H}_{16} \mathrm{ClN}_{5} \mathrm{O}_{2}$ : C, 64.26; H. 3.75; N. 16.29; Found: C. 64.25; H. 3.73; N. 16.24.

5-Bromo-2'-phenyl-6' H-spiro[indole-3,5' $[1,2,4]$ triazolo[1,5-c]quinazoline]-2(1H)-one (3g). Yield - 88.7\%. M. p. - 291-293 ${ }^{\circ} \mathrm{C}$; IR, v, $\mathrm{cm}^{-1}: 3173$, 3130, 3094, 2966, 2940, 2920, 2853, 1734, 1681, 1620, $1593,1544,1517,1478,1441,1400,1345,1318,1282$, $1263,1195,1180,1150,1128,1106,1068,1059,1026$, 1005, 974, 937, 921, 888, 874, 847, 820, 786, 768, 743, 722, 699, 687, 635; ${ }^{1} \mathrm{H}$ NMR, $\delta$, ppm. (J, Hz): 10.68 (s, $1 \mathrm{H}, \mathrm{NH}$ indol), 8.01 (d, $J=7.4 \mathrm{~Hz}, 2 \mathrm{H}, \mathrm{H}-2,6 \mathrm{Ph}), 7.84$ (d, $J=7.4$ Hz, 1H, H-10), 7.75 (s, 1H, NH quin.), 7.607.45 (m, 2H, H-4,6 indol), 7.43-7.29 (m, 3H, H-3,4,5 Ph), 7.24 (t, $J=7.4 \mathrm{~Hz}, 1 \mathrm{H}, \mathrm{H}-8), 6.94$ (d, $J=8.8 \mathrm{~Hz}, 1 \mathrm{H}$, H-7), 6.90-6.78 (m, 2H, H-7 indol, H-9); LC-MS, m/z = 444.0 [M] ${ }^{+}$; Anal. Calcd for $\mathrm{C}_{22} \mathrm{H}_{14} \mathrm{BrN}_{5} \mathrm{O}: \mathrm{C}, 59.47$; H. 3.18; N. 15.76; Found: C. 59.46; H. 3.14; N. 15.72.

5-Bromo-2'-(4-fluorophenyl)-6'H-spiro[indole3,5'-[1,2,4]triazolo[1,5-c] quinazoline]-2(1H)one (3h). Yield - 86.5\%. M. p. $-266-268^{\circ} \mathrm{C}$; IR, v, $\mathrm{cm}^{-1}$ : 3174, 3101, 2950, 2857, 2787, 1732, 1616, 1545, 1516, $1479,1470,1446,1417,1360,1338,1314,1290,1264$, $1233,1222,1197,1184,1152,1126,1108,1092,1061$, $1012,975,941,900,874,844,820,784,764,749$, 711, 699, 683, 665, 636, 613; ${ }^{1} \mathrm{H}$ NMR, $\delta$, ppm. (J, Hz): 
10.68 (s, 1H, NH indol), 8.10-7.99 (m, 2H, H-2,6 Ph), 7.81 (d, $J=7.5 \mathrm{~Hz}, 1 \mathrm{H}, \mathrm{H}-10), 7.76$ (s, 1H, NH quin.), 7.58-7.47 (m, 2H, H-4,6 indol), $7.25(\mathrm{~m}, 1 \mathrm{H}, \mathrm{H}-8)$, $7.10(\mathrm{t}, J=8.6 \mathrm{~Hz}, 2 \mathrm{H}, \mathrm{H}-3,5 \mathrm{Ph}), 6.94(\mathrm{~d}, J=8.5 \mathrm{~Hz}$, 1H, H-7), 6.90-6.77 (m, 2H, H-9, H-7 indol); LC-MS, $\mathrm{m} / \mathrm{z}=464.0[\mathrm{M}+2]$; Anal. Calcd for $\mathrm{C}_{22} \mathrm{H}_{13} \mathrm{BrFN}_{5} \mathrm{O}: \mathrm{C}$, 57.16; H. 2.83; N. 15.15; Found: C. 57.15; H. 2.85; N. 15.17 .

5-Bromo-2'-(4-bromophenyl)-6' H-spiro[indole$3,5^{\prime}-[1,2,4]$ triazolo [1,5-c]quinazoline $]-2(1 \mathrm{H})$ one (3i). Yield $-81.2 \%$. M. p. $-219-221^{\circ} \mathrm{C}$; IR, $v, \mathrm{~cm}^{-1}$ : $3174,3093,3075,3046,2991,2917,2836,2718,2678$, $2533,2351,1748,1705,1680,1612,1586,1516,1469$, $1445,1426,1399,1318,1292,1271,1237,1208,1178$, $1124,1109,1068,1053,1011,972,927,902,890$, $844,807,755,717,698,681,660,636,628$; LC-MS, $\mathrm{m} / \mathrm{z}=524.0[\mathrm{M}]^{+} ;$Anal. Calcd for $\mathrm{C}_{22} \mathrm{H}_{13} \mathrm{Br}_{2} \mathrm{~N}_{5} \mathrm{O}: \mathrm{C}, 50.51$; H. 2.50; N. 13.39; Found: C. 50.55; H. 2.53; N. 13.42.

5-Bromo-2'-(2-methoxyphenyl)-6'H-spiro[indole-3,5'-[1,2,4]triazolo[1,5-c]quinazoline]-2(1H)one (3j). Yield $-71.6 \%$. M. p. $-263-265^{\circ} \mathrm{C}$; IR, $v, \mathrm{~cm}^{-1}$ : $3178,3101,3024,2950,2903,2833,1735,1684,1654$, $1616,1602,1577,1547,1514,1473,1436,1420,1388$, $1337,1310,1285,1266,1243,1182,1150,1132,1090$, $1056,1023,970,935,888,870,830,818,741,713$, $700,678,667,635,624,608$; ${ }^{1} \mathrm{H}$ NMR, $\delta$, ppm. $(J, \mathrm{~Hz})$ : 10.67 (s, $1 \mathrm{H}, \mathrm{NH}$ indol), 7.83 (d, $J=7.3 \mathrm{~Hz}, 1 \mathrm{H}, \mathrm{H}-10$ ), 7.75 (s, $1 \mathrm{H}, \mathrm{NH}$ quin.), 7.67 (d, $J=7.3 \mathrm{~Hz}, 1 \mathrm{H}, \mathrm{H}-6 \mathrm{Ph})$, 7.59-7.47 (m, 2H, H-4,6 indol), $7.34(\mathrm{t}, J=7.3 \mathrm{~Hz}, 1 \mathrm{H}$, $\mathrm{H}-8), 7.24(\mathrm{t}, J=7.2 \mathrm{~Hz}, 1 \mathrm{H}, \mathrm{H}-9), 7.06-6.89(\mathrm{~m}, 3 \mathrm{H}$, H-7 indol, H-4 Ph, H-7), 6.89-6.79 (m, 2H, H-3,5 Ph), 3.78 (s, $3 \mathrm{H},-\mathrm{OCH}_{3}$ ); LC-MS, $\mathrm{m} / \mathrm{z}=474.0[\mathrm{M}]^{+}$; Anal. Calcd for $\mathrm{C}_{23} \mathrm{H}_{16} \mathrm{BrN}_{5} \mathrm{O}_{2}$ : C. 58.24; H. 3.40; N. 14.77; Found: C. 58.20; H. 3.39; N. 14.75.

$\mathrm{N}$-(3-Methoxybenzyl)-2-[2'-(3-fluorophenyl)-2-oxo-6'H-spiro[indole-3,5'-[1,2,4] triazolo[1,5-c]quinazoline]-1(2H)-yl]acetamide (3k). Yield - 98.11\%. M. p. - 152-154 ${ }^{\circ} \mathrm{C}$; IR, v, $\mathrm{cm}^{-1}: 3314$, $3298,3077,3000,2923,2848,1733,1678,1616,1588$, $1516,1505,1490,1470,1417,1360,1326,1261,1215$, $1204,1176,1155,1107,1071,1035,1010,963,924$, $874,804,790,744,696,680,665,633,622 ;{ }^{1} \mathrm{H}$ NMR, $\delta$, ppm. (J, Hz): 8.55 (bst, $1 \mathrm{H},-\mathrm{CON} \underline{\mathrm{H}} \mathrm{CH}_{2}-$ ), 7.94-7.84 (m, 2H, NH quin., H-6 3-FPh), 7.80 (d, J= 7.5 Hz, $1 \mathrm{H}$, $\mathrm{H}-10$ ), 7.69 (d, $J=9.7 \mathrm{~Hz}, 1 \mathrm{H}, \mathrm{H}-2 \mathrm{3}-\mathrm{FPh}), 7.58-7.44$ (m, 2H, H-4,6 indol), 7.39 (dd, $J=13.7,6.2 \mathrm{~Hz}, 1 \mathrm{H}$, H-5 3-FPh), 7.27 (t, J = 7.4 Hz, 1H, H-8), 7.19 (m, 2H, H-4 3-FPh, H-9), 7.15-7.04 (m, 2H, H-5,7 indol), 6.97$6.86\left(\mathrm{~m}, 2 \mathrm{H}, \mathrm{H}-5 \mathrm{3}-\mathrm{CH}_{3} \mathrm{OPh}\right), 6.86-6.78(\mathrm{~m}, 2 \mathrm{H}, \mathrm{H}-2,6$ $\left.3-\mathrm{CH}_{3} \mathrm{OPh}\right), 6.74\left(\mathrm{~d}, J=7.9 \mathrm{~Hz}, 1 \mathrm{H}, \mathrm{H}-43-\mathrm{CH}_{3} \mathrm{OPh}\right.$ ), $4.50\left(\mathrm{~d}, J=16.1 \mathrm{~Hz}, 1 \mathrm{H},-\mathrm{CH}_{2} \mathrm{CO}-\right), 4.43-4.25(\mathrm{~m}, 2 \mathrm{H}$, $-\mathrm{CH}_{2} \mathrm{CO}-,-\mathrm{NHCH}_{2}-$ ), 3.73 (s, $1 \mathrm{H}, \mathrm{CH}_{3} \mathrm{O}-$ ); LC-MS, m/z = $561.2[\mathrm{M}+1]$; Anal. Calcd for $\mathrm{C}_{32} \mathrm{H}_{25} \mathrm{FN}_{6} \mathrm{O}_{3}$ : C, 68.56; H. 4.50; N. 14.99; Found: C. 68.59; H. 4.57; N. 15.02.
N-(4-Methoxyphenyl)-2-[2'-(4-methoxyphenyl)-2-oxo-6'H-spiro[indole-3, 5'- $[1,2,4]$ triazolo[1,5-c]quinazoline]-1(2H)-yl]acetamide (3l). Yield - 49.3\%. M. p. $-214-216^{\circ} \mathrm{C}$; IR, v, $\mathrm{cm}^{-1}: 2919$, $2851,1732,1681,1652,1612,1544,1509,1489,1466$, $1455,1437,1418,1377,1360,1301,1239,1170,1107$, $1029,1010,965,864,829,785,752,704,683,664$, 638; ${ }^{1} \mathrm{H}$ NMR, $\delta$, ppm. (J, Hz): 10.07 (s, 1H, -NHCO-), 7.90 (d, J= 7.8 Hz, 1H, H-2,6 2'- $\mathrm{Ph}$ ), 7.88-7.76 (m, 2H, $\mathrm{H}-10$, NH quin.), 7.66-7.41 (m, 4H, H-4,6 indol, $\mathrm{H}-2,6$ $\mathrm{Ph}), 7.24(\mathrm{t}, J=7.5 \mathrm{~Hz}, 1 \mathrm{H}, \mathrm{H}-8), 7.18(\mathrm{t}, J=7.2 \mathrm{~Hz}, 1 \mathrm{H}$, $\mathrm{H}-9$ ), 7.13 (d, $J=7.5 \mathrm{~Hz}, 1 \mathrm{H}, \mathrm{H}-7), 6.97-6.74\left(\mathrm{~m}, 6^{\prime} \mathrm{H}\right.$, H-5,7-indol, H-3,5 2'-Ph, H-3,5 Ph), 4.60 (d, $J=17.1$ $\mathrm{Hz}, 1 \mathrm{H},-\mathrm{CH}_{2}-$ ) $, 4.45\left(\mathrm{t}, J=16.3 \mathrm{~Hz}, 1 \mathrm{H},-\mathrm{CH}_{2}-\right), 3.81(\mathrm{~s}$, $\left.1 \mathrm{H},-\mathrm{OCH}_{3}\right), 3.75\left(\mathrm{~s}, 1 \mathrm{H},-\mathrm{OCH}_{3}\right) ; \mathrm{LC}-\mathrm{MS}, \mathrm{m} / \mathrm{z}=559.0$ $[\mathrm{M}+1]$; Anal. Calcd for $\mathrm{C}_{32} \mathrm{H}_{26} \mathrm{~N}_{6} \mathrm{O}_{4}: \mathrm{C}, 68.81 ;$ H. 4.69; N. 15.04; Found: C. 68.84; H. 4.73; N. 15.09.

\section{$\mathrm{X}$-ray Experimental Part}

The colourless crystals of $\mathbf{2 a}\left(\mathrm{C}_{19} \mathrm{H}_{18} \mathrm{~N}_{4}\right)$ are monoclinic. At $293 \mathrm{~K} \mathrm{a}=10.4412(7), \mathrm{b}=14.6803(6), \mathrm{c}=$ 10.7776(5) $\AA, \beta=110.913(6)^{\circ}, V=1543.2(1) \AA^{3}, M_{r}=$ $302.37, \mathrm{Z}=4$, space group $\mathrm{P} 2_{1} / \mathrm{n}, \mathrm{d}_{\text {calc }}=1.301 \mathrm{~g} / \mathrm{cm}^{3}$, $\mu\left(\mathrm{MoK}_{\alpha}\right)=0.080 \mathrm{~mm}^{-1}, \mathrm{~F}(000)=640$. Intensities of 15494 reflections (4500 independent, $\mathrm{R}_{\mathrm{int}}=0.024$ ) were measured on a "Xcalibur-3" diffractometer (graphite monochromated $\mathrm{MoK}_{\alpha}$ radiation, CCD detector, $\omega$-scaning, $2 \Theta_{\max }=60^{\circ}$ ). The structure was solved by the direct method using SHELXTL package [17]. The restrictions on the bond lengths of the disordered part $\left(C s p^{3}-C_{s} p^{3} 1.54 \AA\right.$ ) $)$ were applied. Positions of the hydrogen atoms were located from the electron density difference maps and refined by a "riding" model with $U_{\text {iso }}=1.2 U_{\text {eq }}$ of the carrier atom. The hydrogen atom of the amino group was refined using isotropic approximation. Full-matrix least-squares refinement against $\mathrm{F}^{2}$ in anisotropic approximation for non-hydrogen atoms using 4410 reflections was converged to $\mathrm{wR}_{2}=0.150\left(\mathrm{R}_{1}=0.052\right.$ for 3137 reflections with $F>4 \sigma(F), S=1.012)$. The final atomic coordinates, and crystallographic data for molecule $\mathbf{2 a}$ were deposited to with the Cambridge Crystallographic Data Centre, 12 Union Road, CB2 1EZ, UK (fax: +44-1223336033; e-mail: deposit@ccdc.cam.ac.uk). They are available on request quoting the deposition numbers CCDC 1408959.

\section{Conclusions}

2'-Aryl-6' $H$-spiro[1,2,4]triazolo[1,5-c] quinazolines have been obtained by [5+1]-cyclocondensation of [2-(3-aryl-1H-1,2,4-triazolo-5-yl)phenyl]amines and carbonyl compounds. The spectral characteristics of the compounds $\left({ }^{1} \mathrm{H}\right.$ and ${ }^{13} \mathrm{C}$ NMR, mass spectrometry and X-ray analysis) have been discussed. 


\section{References}

1. Amin K. M., Anwar M. M., Syam Y. M., Khedr M., Kamel M. M., Kassem E. M. M. A. novel class of substituted spiro[quinazoline-2,1'-cyclohexane] derivatives as effective PPAR-1 inhibitors: molecular modeling, synthesis, cytotoxic and enzyme assay evaluation. Acta. Pol. Pharm. Drug. Res., 2013, Vol. 70(4), pp.687-708.

2. Mustazza C., Borioni A., Sestili I., Sbraccia M., Rodomonte A., Ferretti R., Rosaria Del Giudice M. Synthesis and evaluation as NOP ligands of some spiro[piperidine4,2(1H)-quinazolin]-4(3H)-ones and spiro[piperidine-4,5(6H)-[1,2,4]triazolo[1,5-c]quinazolines]. Chem. Pharm. Bull., 2006 , Vol. 54(5), pp.611-622.

3. Pat. US 005326765 A. A 61 K 31/505, C 07 D 403/04. 2,2,4-Trialkyl-1,2-dihydroquinazoline-3-oxides / D. W. Combs, R. Falotico, V. Bandurco (US); Ortho Pharmaceutical Corporation; Filed: 06.11.1991. Posted: 05.06.1994.

4. Bunce R. A., Nammalwar B. New conditions for synthesis of (6)-2-monosubstituted and (6)-2,2-disubstituted 2,3-dihydro-4(1H)-quinazolinones from 2-nitro- and 2-aminobenzamide. J. Heterocyclic Chem., 2011, Vol. 48, pp.991-997.

5. Lessel J. 1,2-Dihydro-4-chinazolinone aus anthranilamiden und oxoverbindungen - Untersuchungen zum Reaktionsverlauf der Ringschlufireaktion. Arch. Pharm., 1994, Vol. 327, pp.571-579.

6. Karimi-Jaberi Z., Zarei L. Rapid synthesis of 2-substituted-2,3-dihydro-4(1H)-quinazolinones using boric acid or sodium dihydrogen phosphate under solvent-free conditions. S. Afr. J. Chem., 2012, Vol. 65, pp.36-38.

7. Klemm L. H., Weakley T. J. R., Gilbertson R. D., Song Y. H. Definitive structural assignment of condensation products from anthranilamides and 3-amino-2-carbamoylthiphene with keones. Formation of tetrahydroquinazolines and their thiophene isosters. J. Heterocyclic. Chem., 1998, Vol. 35 , p.1269.

8. Miklós F., Fülöp F. "Dry" and "wet" green synthesis of 2,2-disubstituted quinazolinones. Eur. J. Org. Chem., 2010, pp.959-965.

9. Karimi-Jaberi Z., Zarei L. Tris(hydrogensulfato)boron catalysed rapid synthesis of 2-substituted-2,3-dihydroquinazolin-4(1H)-ones under solventfree conditions. J. Chem. Res., 2012, pp.194-196.

10. Lessel J. 3,1-Benzoxazine und tetrahydrochinazoline aus o-aminobenzylalkohol und o-aminobenzylamin - semi-empirische Mo-berechnungen zum cyclisierungsverhalten. Arch. Pharm. (Weinheim), 1994, Vol. 327, pp.329-336.

11. Kempter G., Ehrlichmann W., Plesse M., Lehm H. U. 1,3-Unsubstituierte 1,2,3,4-tetrahydrochinazoline aus 1,3-diaminen. J. Prakt. Chemie., 1982, Vol. 334 (5), pp.832-840.

12. Kovalenko S. I., Voloshina V. O., Karpenko O. V. Synthesis and nucleophilic cleavage of 2-R-[1,2,4]triazolo[1,5-c]quinazoline. J. Org. Pharm. Chem., 2010, Vol. 8 (2(30), pp.48-56.

13. Breitmaier E. Structure Elucidation by NMR in Organic Chemistry: A Practical Guide / E. Breitmaier. - [3-rd Revised Ed.]. - Wiley, 2002, p.272.

14. Kovalenko S. I., Antypenko L. M., Bilyi A. K., Kholodnyak S. V., Karpenko O. V., Antypenko O. M., Mykhaylova N. S., Los' T. I., Kolomoets' O. S. Synthesis and anticancer activity of 2-alkyl(alkaryl-, aryl-, heteryl-)-[1,2,4]triazolo[1,5-c]quinazolines. Sci. Pharm., 2013, Vol. 81(2), pp.359-391.

15. Zephyrov Yu. V. Reduced intermolecular contacts and specific interactions in molecular crystals. Crystallography., 1997, Vol. 42, p.936.

16. Ward C. S. The Chemistry of Isatin. Chem. Rev., 1944, Vol. 34(3), pp.393-434.

17. Sheldrick G. M. A short history of SHELX. Acta Crystallogr., Sect. A, 2008, Vol. 64(1), pp.112-122.

Надійшла до редакції 22.07.2016 р.

\section{Acknowledgements}

The authors gratefully acknowledge "Enamine Ltd." (Kyiv, Ukraine) for financial support of this work. The authors have declared no conflict of interest. 